\title{
Study of Scintillation, Fluorescence and Scattering in Mineral Oil for the MiniBooNE Neutrino Detector
}

\author{
Bruce C. Brown, Senior-Member, IEEE,Stephen Brice, Eric Hawker, Shannon Maza, Hans-Otto Meyer, \\ Anna Pla-Dalmau, Rex Tayloe, Hirohisa A. Tanaka and Dmitri Toptygin
}

\begin{abstract}
The MiniBooNE neutrino detector at Fermilab (FNAL) is filled with 250,000 gallons of pure mineral oil. The principal signal for MiniBooNE is light observed in a prompt Cherenkov cone. Scattering and fluorescence modify our detection of this light. Scintillation is also created by ionization in the oil. Studies of fluorescence of this oil have been carried out over a wide spectrum of exciting light and time resolved fluorescence with a narrower range of excitation. Polarized scattering measurements have been carried out at longer wavelengths. Time resolved and spectrally resolved scintillation has been studied with a $200 \mathrm{MeV}$ Proton beam at the Indiana University Cyclotron Facility. Results of these studies will be reported.
\end{abstract}

Index Terms-Cherenkov, light extinction, fluorescence, scintillation.

\section{INTRODUCTION}

$\mathbf{T}$ HE MiniBooNE Experiment at Fermilab (E898) detects neutrino interactions in a $12 \mathrm{~m}$ diameter sphere of pure (undoped) mineral oil. The beam is created by the decay of mesons which are produced by the interaction of $8 \mathrm{GeV}$ protons from the Fermilab Booster Accelerator. The mesons are focused by a magnetic horn into a $50 \mathrm{~m}$ decay pipe where they decay to produce an nearly pure muon-neutrino beam. Most of the detected neutrinos have energies 0.2 to $2.0 \mathrm{GeV}$. The principal goal is to detect electron-neutrino interactions which may result from the oscillation of the muon-neutrinos into electron-neutrinos in the $500 \mathrm{~m}$ from the target to the detector.

Interactions with the carbon nucleus or protons in the oil produce charged particles (muons, electrons, pions...) which will produce Cherenkov light if they have sufficient velocity (energy). The cone of light produced by a track segment projects to a well-defined pattern on the photomultiplier array. They will arrive at the photomultiplier with only the delay due to the velocity of light in the oil. Some Cherenkov light will be lost to scattering, absorption by fluorescent molecules or absorption by other processes, modifying the resulting pattern of observed light signals. In addition, the ionization produced

Manuscript received October 20, 2004.

Bruce C. Brown, Stephen Brice, Shannon Maza, and Anna Pla-Dalmau are with the Fermi National Accelerator Laboratory which is operated by Universities Research Association Inc. under Contract No. DE-AC02-76CH03000 with the United States Department of Energy. Eric Hawker is with the Physics Department of Western Illinois University. Hans-Otto Meyer and Rex Tayloe are with the Physics Department of Indiana University. Hirohisa A. Tanaka is with the Physics Department of Princeton University. Dmitri Toptygin is with the Biology Department at Johns Hopkins University.

\begin{tabular}{lccc}
\hline \hline \multirow{2}{*}{ Wavelength } & Theory & Total & Isotropic \\
\hline $442 \mathrm{~nm}$ & $62.3 \pm 1.8$ & $51.7 \pm 7.0$ & $63.5 \pm 7.0$ \\
$532 \mathrm{~nm}$ & $136.5 \pm 3.9$ & $114.5 \pm 15.4$ & $136.3 \pm 15.4$ \\
\hline \multicolumn{4}{c}{ TABLE I } \\
COMPARISON OF EXPECTED SCATTERING LENGTHS FROM THE \\
INSTEIN-SMOLUCHOWSKI EQUATION AND MEASURED VALUES. THE \\
“ISOTROPIC” CONTRIBUTION IS OBTAINED BY SUBTRACTING THE \\
APPROPRIATE ANISOTROPIC CONTRIBUTION FROM THE TOTAL.
\end{tabular}

by charged particles (including those which produce Cherenkov light) will result in scintillation which will also propagate to the detectors. The scattered light, re-emitted fluorescent light and scintillation will be delayed by path length and excited state lifetimes so that it will arrive with some delay. Geometrically, fluorescence and scintillation radiate isotropically from their source.

The oil for the MiniBooNE detector was selected after extensive tests[1]. It was shipped to Fermilab in rail tank cars. Transfer of the oil to the detector was completed in May 2002. The oil for the tests reported in the paper was extracted from the detector.

\section{SCATTERING}

$\mathbf{S}$ CATTERING from the density fluctuations in oil provide an inescapable limit on the transmission of the light. Elementary arguments demonstrate that this process accounts for an attenuation length which rises as $\lambda^{4}$. Scattering measurements have been carried out at two wavelengths. To predict this scattering, measurements of the compressibility of the oil were performed[2].

\section{A. Scattering Measurements with a Goniometer}

Studies of light scattering in Marcol 7 mineral oil measured scattering at $442 \mathrm{~nm}$ and $532 \mathrm{~nm}$ incident wavelength using a Brookhaven BIS-200 goniometer[3] at the Chemical Engineering Department at Princeton University. Fig 1 describes the measurements and results. The angular distribution of light was recorded for both polarizations and each wavelength. Data for the $442 \mathrm{~nm}$ laser are shown in fig 1(a) along with fits. The strengths of isotropic to anisotropic terms for the scattering tensor are in a ratio of 1:0.09. Results for these scattering measurement are shown in Table I. 


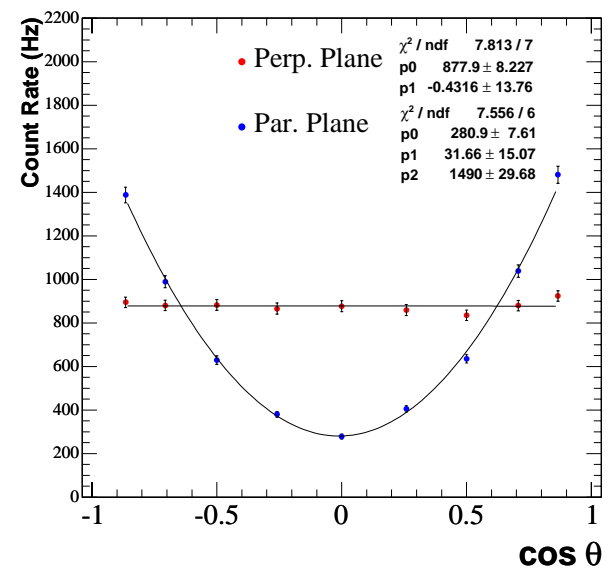

(a) Scattering Angular Distribution

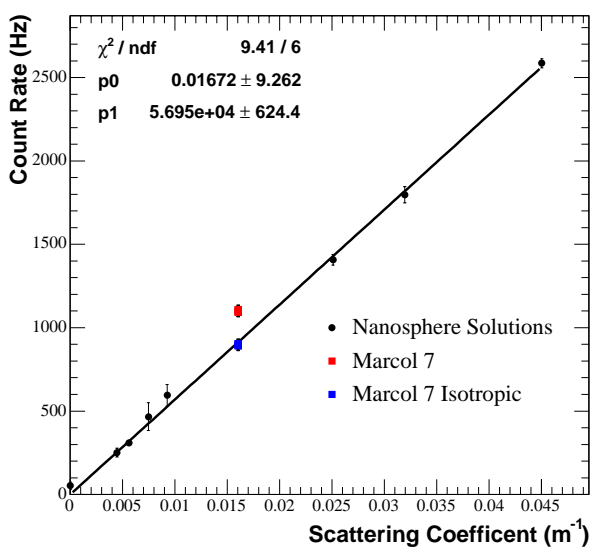

(b) Scattering Rate

Fig. 1. Light scattered in Marcol 7 is measured using a BIS goniometer and $442 \mathrm{~nm}$ laser. Incident light is linearly polarized. Angular dependence was measured for angles between $\theta=25^{\circ}$ and $155^{\circ}$ from the incident beam. For the scattering angular distribution, the red points are measured in plane perpendicular to the incident polarization, while the blue points are measured in the plane parallel to the incident polarization. The former are fit to a first order polynomial, while the latter are fit to a second order polynomial. The distributions are consistent with Rayleigh scattering off isotropic density perturbations in the mineral oil with an additional contribution from non-isotropic fluctuations. The scattering rate plot shows the intensity of light scattered off suspensions of $50 \mathrm{~nm}$ polysterene nanospheres in water (black points) and Marcol 7 (red and blue points) The different black points correspond to different concentrations of nanospheres, with the abscissas placed according to the predictions of the Mie expansion. The black points have a subtraction corresponding to Rayleigh scattering in the water itself and are fit to a first-order polynomial to determine the Rate-to-scattering coefficient conversion factor. The red point is the total observed scattering in Marcol 7, while the blue point results from isolating the contribution from isotropic density fluctuations from the non-isotropic component using the measured angular distributions. The abscissa for these two points are at the scattering coefficient predicted by the Einstein-Smoluchowski equation.
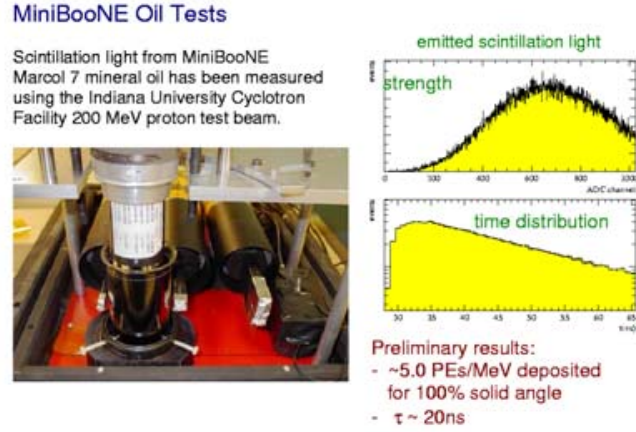

Fig. 2. Preliminary results from IUCF Scintillation Tests

The spectrofluorometer also records Rayleigh and Raman scattering. An extensive emission/excitation matrix was recorded with the Fermilab instrument. The configuration recorded the spectra at $90^{\circ}$ from the excitation. Rayleigh scattering signals were isolated at wavelengths from 320 to 460 $\mathrm{nm}$ and the expected wavelength dependence was observed. A signal due to Raman scattering was clearly observed and contributes a few percent of additional scattering.

\section{SCINTILLATION}

$\mathbf{T}$ HE MiniBooNE collaboration planned to employ scintillation signals to add information which would help identify the various neutrino-induced interactions since some processes will produce additional slow-moving hadronic particles. In order to begin understanding the scintillation processes, a test of scintillation light produced by the ionization loss of $200 \mathrm{Mev}$

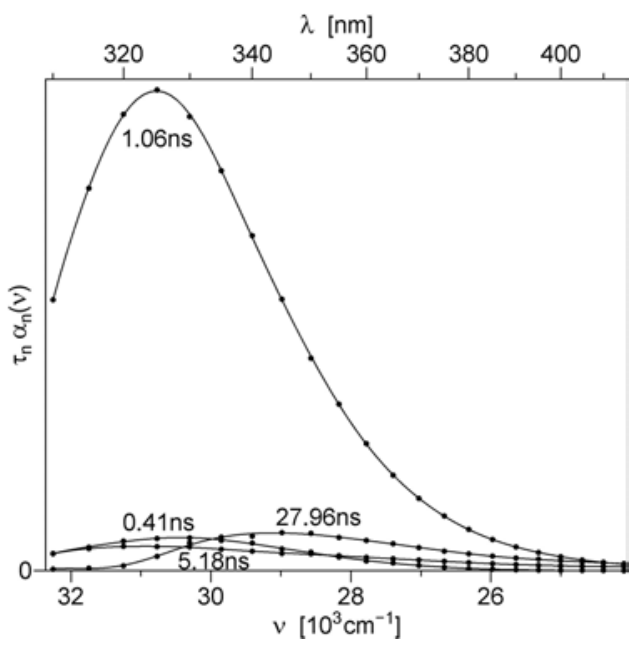

Fig. 3. Decay Associated Spectrum of $123 \mu \mathrm{M}$ solution of Vitamin $\mathrm{E}$ in Marcol 7.

protons ( $v$ below Cherenkov threshold) in samples of Marol 7 oil from MiniBooNE was conducted at the Indiana University Cyclotron Facility. Some initial results are shown in fig 2. Time distributions show scintillation components with time constants $\sim 20$ ns. Time and wavelength spectral measurements of the scintillation are ongoing.

\section{FLuORESCENCE}

$\mathbf{T}$ HE observation of scintillation in this oil encouraged the MiniBooNE collaborators to contact the Scintillation 

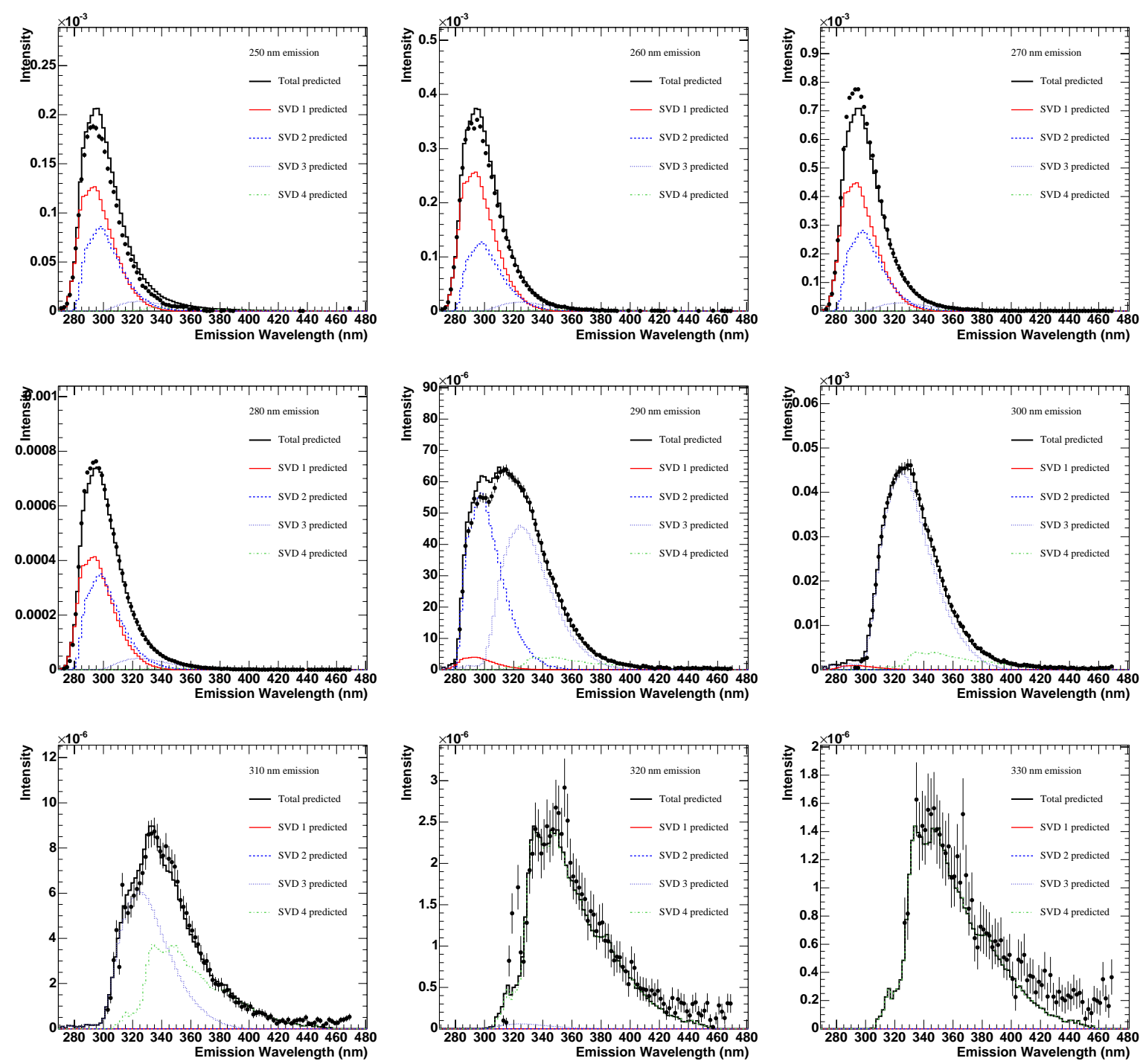

Fig. 4. Comparison of background and scattering-subtracted emission curves (black points) with emission predicted by the SVD analysis (black line). Data for nine excitation wavelengths are shown. The expected contribution from each fluorophore is shown by the colored lines (red, blue, violet and green for fluorophores $1,2,3$ and 4 , respectively).

Detector Development Group (SDDG, A. P-D, leader) at Fermilab. Fluorescence was measured in the spectrofluorometer at Fermilab and strong signals were seen. As a result, a collaboration was formed to measure the time resolved signal from this fluorescence using facilities at the Biology Department at Johns Hopkins University. Subsequently measurements have been carried out at both laboratories to examine the details of the fluorescence process.

\section{A. Details on Vitamin E Fluorescence}

The vendor added Vitamin E ( $\alpha$-tocopherol) to Marcol 7 as an antioxidant. One initial study was to explore the fluorescent contribution of this additive. Absorbance measurements on solutions prepared by serial dilution, and excitation and emission spectra with and without added Vitamin E provided spectra of this fluor. Time resolved fluorescence measurements using 300 $\mathrm{nm}$ laser excitation were perfomed on the $123 \mu \mathrm{M}$ solution. The emission monochromator was set to 22 different wavelengths between $315 \mathrm{~nm}$ and $415 \mathrm{~nm}$, with $5 \mathrm{~nm}$ intervals. At each of the 22 wavelengths two decay curves were measured: one with vitamin $\mathrm{E}$ solution and one with the solvent alone. A third cuvette contained a scatterer (colloidal silica in water) and was used to measure the instrument response function. A global analysis (see below) of this data produced the DecayAssociated Spectra shown in fig 3 for the sample with enhanced Vitamin E. Comparison with the similar plot for the oil alone indicates that the term with a $1.06 \mathrm{~ns}$ decay time is due to the Vitamin E. The term with $0.41 \mathrm{~ns}$ time constant is also enhanced in the sample with enhanced Vitamin E. From the extinction coefficient spectrum and the fluorescence emission spectrum one can estimate the radiative decay rate (inverse of 
the natural lifetime) with the Strickler and Berg equation[4]. This estimate is $\tau_{0}=19.6 \mathrm{~ns}$ for the natural lifetime. From this we extimate the quantum yield of fluorescence as $\tau / \tau_{0}=5.4 \%$

\section{B. Fluorescence Emission and Excitation and SVD Analysis}

At JHU, excitation and emission fluorescent spectra were recorded in a matrix of wavelengths (106 rows and 52 columns). The emission wavelength range for the excitation spectra was from $270 \mathrm{~nm}$ to $480 \mathrm{~nm}$, with a $2 \mathrm{~nm}$ spacing between data points (106 point total). The 52 emission spectra included measurements with excitation from $250 \mathrm{~nm}$ to $340 \mathrm{~nm}$ with spacing of $2 \mathrm{~nm}$ (46 points) and from $340 \mathrm{~nm}$ to $370 \mathrm{~nm}$ with spacing of $5 \mathrm{~nm}$ ( 6 additional points, 52 points total). To obtain fluorescence signals, Singular Value Decomposition (SVD) was performed on completely uncorrected data[5]. Spectral features due to Rayleigh or Raman scattered light were eliminated by identifying them and replacing their contribution iteratively. Four singular vectors were identified with significant signal above backgroud. Linear combinations of the column singular vectors represent the emission spectra of four hypothetical fluorescent species while linear combinations of the row singular vectors represent the excitation spectra. To obtain spectra which are (dominantly) non-negative, a transform is required. The matrix $\mathbf{R}$ transforms the singular column vectors to the emission spectra; matrix $\mathbf{Q}$ transforms the singular row vectors to the excitation spectra; the product $\mathbf{Q R}$ is the diagonal matrix of the singular values. The freedom of choice of the elements of matrices $\mathbf{Q}$ and $\mathbf{Q R}$ was used to make the spectra (largely) nonnegative. Instrumental corrections were applied to the resulting spectra.

In fig 4, we show some results from an independent emissionexcitation matrix recorded at FNAL. Scattering features were fit and removed from the fluorescence spectra and corrections applied for instrumental sensitivity. Nine of the resulting emission spectra are plotted along with the single fluor emission curves and their sum from the JHU SVD analysis.

\section{Time Resolved Fluorescence Measurements}

A sample of Marcol 7 Oil, extracted from the MiniBooNE experiment, in a $1 \mathrm{~cm} \times 1 \mathrm{~cm}$ quartz cuvette was excited by a 11 picosecond pulse from a $285 \mathrm{~nm}$ dye laser. The polarization of the exciting radiation was vertical. Fluorescence emission was collected through a polarizer set to $55^{\circ}$ from the vertical (the magic angle) and a monochromator with 8 $\mathrm{nm}$ bandpass. Fluorescence decay curves were recorded at 32 emission wavelengths in $5 \mathrm{~nm}$ steps from $295 \mathrm{~nm}$ to $450 \mathrm{~nm}$. A global fit using a sum of five exponential terms, $\alpha_{n} * \exp \left(-t / \tau_{n}\right)$ plus additional terms for known instrumental errors which was then convoluted with the experimentallymeasured instrument response function. The convolution was then fitted to the experimental data. The results are displayed as pre-exponential spectra, $\alpha_{n}(\lambda)$ (fig 5) or as the decay-associated spectra, $\alpha_{n}(\lambda) \tau_{n}$ (fig 6). Points represent the parameter values evaluated by the global fitting algorithm. Error bars represent

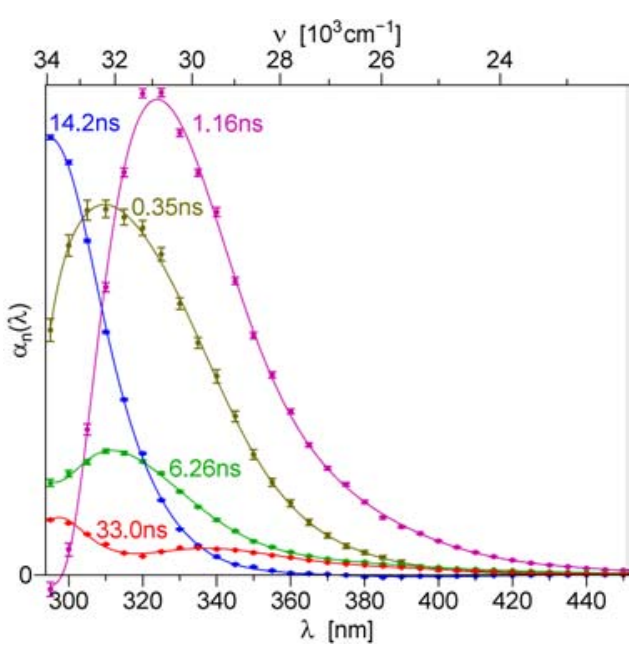

Fig. 5. Spectra of pre-exponential factors from Time-Resolved Emission Spectra recorded with $285 \mathrm{~nm}$ excitations.

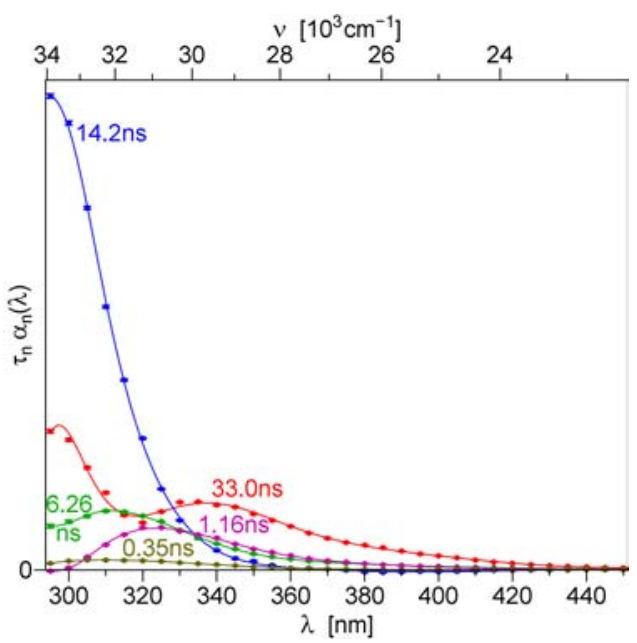

Fig. 6. Decay-associated Spectra from Time-Resolved Emission Spectra recorded with $285 \mathrm{~nm}$ excitations.

90\% confidence intervals. Continuous lines are polynomial fits to the points, for further detail see [5]. Decay-associated spectra represent the contributions of individual fluorophores to the steady-state fluorescence intensity.

Examining these spectra we observe that the $1.16 \mathrm{~ns}$ dominates the emission at early times and matches well the emission spectrum measured for Vitamin E. The components fit with lifetimes of 14.24 and 33.03 ns will be very important for MiniBooNE.

\section{Extinction Measurements}

$\mathbf{T}$ HE $1.6 \mathrm{~m}$ instruments used for the selection of the oil[1] provided the extinction (attenuation) of the oil at 460 $\mathrm{nm}$ with the variable pathlength system and as a function of wavelength with a fixed path in the other instrument. Measurements were limited to wavelengths above the cutoff of the glass window at about $310 \mathrm{~nm}$. To extend the range, measurements were carried out at Fermilab and JHU. 
Extinction Rate for MiniBooNE Marcol 7 Mineral Oil

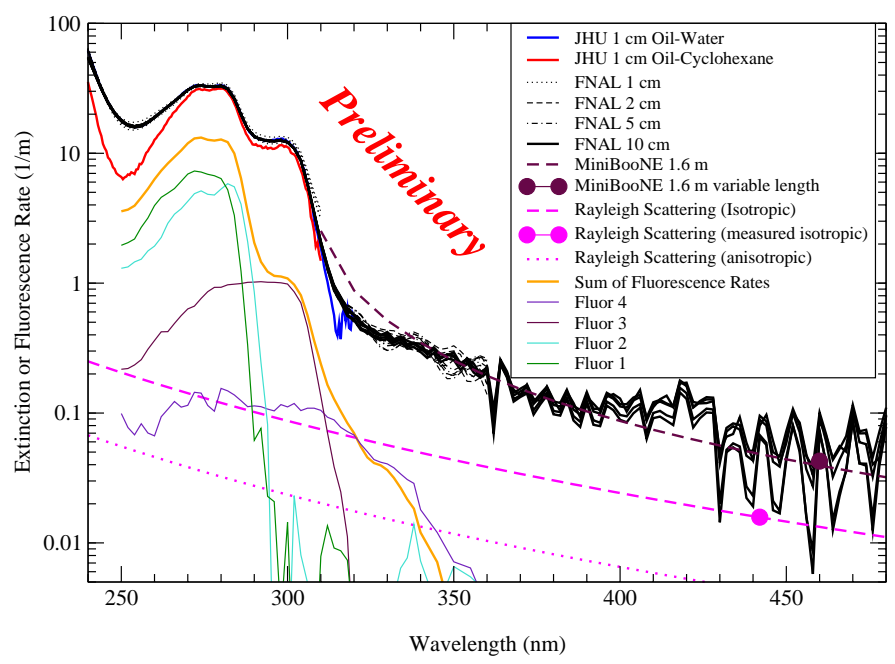

Fig. 7. Measurements at JHU comparing transmission with that of water (blue) or cyclohexane (red) in a $1 \mathrm{~cm}$ cell are converted to extinction. Measurements (five per cell) at Fermilab comparing transmission of Marcol 7 with air (empty cell) were corrected for (wavelength-dependent) reflection and beam divergence effects and converted to extinction rate. Results for quartz cells of $1 \mathrm{~cm}(<310$ $\mathrm{nm}), 2 \mathrm{~cm}(<360 \mathrm{~nm}), 5 \mathrm{~cm}(<360 \mathrm{~nm})$, and $10 \mathrm{~cm}$ are shown with black lines of various styles. The maroon dot shows the result from the MiniBooNE $460 \mathrm{~nm}$ variable path length instrument. The dashed maroon line uses data from the MiniBooNE $1.6 \mathrm{~m}$ variable wavelength instrument normalized to the maroon dot. The magenta dot shows the results of scattering measurements at Princeton $U$ for the isotropic component of Rayleigh scattering which agree with predictions based on the measured compressibility of Marcol 7. The dashed magenta line shows the predicted rate for the isotropic component normalized to the measured point. The anisotropic component is measure to provide a scattering rate of $27 \%$ of the isotropic rate. The magenta dots show the wavelength dependence of that component assuming that the ratio is wavelength independent. The excitation spectra from the SVD analysis (above) corrected for the the instrument response and normalized to the quantum yield of para-terphenyl provides the probability of fluorescent emission per excitation photon per meter of path in Marcol 7. Four hypothetical fluorescent species are shown with solid green, turquoise, maroon and indigo lines. The extinction contribution of these components is obtained by dividing these curves by their respective quantum yields. We identify Fluor 3 with Vitamin $\mathrm{E}$ which was introduced at the refinery.

Transmission of Marcol 7 was measured with a HP Diode Spectrophotometer at the Fermilab SDDG. The corrected transmittances $(T)$ were converted to extinction rates $R_{E}=$ $-(\ln T) / L_{A}$, where $L_{A}$ is the absorber length, and plotted in fig 7. The channel-to-channel variations for data above 350 $\mathrm{nm}$ are instrumental effects which can be smoothed for a final result.

Using a $1 \mathrm{~cm}$ cell, transmission measurements were carried out at JHU. Reflection corrections were reduced using a water sample or eliminated using a cyclohexane sample for comparison. The corrected transmission curves were converted to extinction rate and the results plotted on fig 7 . The oil-water result (blue) is not visible for most wavelengths since it is so consistent with the FNAL results. The oil-cyclohexane result (red) differs from the others due to over-correction because of absorption in the cyclohexane below $270 \mathrm{~nm}$.

The results from the SVD analysis of fluorescence (above) provided normalized rates for the excitation of the four fluors.
Since these dominate the extinction rate, we can compare these emission rates per excitation photon per meter of path to the overall extinction rate. The ratio is the quantum efficiency of the fluor. It peaks at about $40 \%$ for near $280 \mathrm{~nm}$.

\section{SUMMARY}

$\mathbf{T}$ HE Cherenkov signal in MiniBooNE is observed by photomultipliers which are from $<1 \mathrm{~m}$ to $\sim 11 \mathrm{~m}$ from the charged particle track. The scattering and absorption of this light will reduce the signal due to the Cherenkov effect. The data in fig 7 provide the information required as input to the fitting and simulation efforts. It is apparent that fluorescence processes will absorb some ultraviolet Cherenkov photons and re-radiate them isotropically at longer wavelengths. A signal from scintillation will also be measured by the phototubes. It will be isotropic about the charged particle track and will exhibit one or more scintillation time constants. The fluors responsible for scintillation and fluorescence may exhibit the same lifetimes and strengths for the two processes but that is not assured. Further studies of the scintillation are required but fits to preliminary data are consistent with the spectrum of fluorescence time constants. The fluorescent absorption accounts for most extinction at wavelengths shorter than 300 to 320 $\mathrm{nm}$. Above that point some fluorescence contribution remains but from about $400 \mathrm{~nm}$, the difference between the measured extinction and the scattering (isotropic and anisotropic Rayleigh scattering and Raman scattering) is not yet independently measured and it accounts for about $70 \%$ of the extinction. In addition to possible further laboratory tests, analysis of calibration data from the cosmic ray muons and muon-decay electrons will be used to measure or set limits on these effects.

\section{ACKNOWLEDGMENT}

The authors would like to thank Wesley Kopacka of the Chemical Engineering Department of Princeton University for assistance with scattering measurements and Ludwig Brand of the Biology Department at Johns Hopkins University for support and encouragment.

\section{REFERENCES}

[1] J. R. et al., "Mineral oil tests for the MiniBooNE detector," IEEE Transactions on Nuclear Science, vol. 49, p. 95, 2002.

[2] H. O. Meyer, "Compressibiliy of marcol 7," Indiana University, MiniBooNE TN 114, February 2004.

[3] A. O. Bazarko, P. D. Meyers, R. B. Patterson, F. C. Shoemaker, and H. A. Tanaka, "Studies of scattering in marcol 7 mineral oil," Princeton University, MiniBooNE TN 144, September 2004.

[4] D. Toptygin, "Effects of the solvent refractive index and its dispersion on the radiative decay rate and extinction coefficient of a fluorescent solute," Journal of Fluorescence, vol. 13, pp. 201-219, 2003.

[5] D. Toptygin, R. S. Savtchenko, N. D. Meadow, and L. Brand, "Homogeneous spectrally- and time-resolved fluorescence emission from singletriptophan mutants of IIA ${ }^{G l c}$ protein," J. Phys. Chem B, vol. 105, pp. 2043-2055, 2001. 